\title{
Data Acquisition Glove for Hand Movement Impairment Rehabilitation
}

\author{
http://dx.doi.org/10.3991/ijoe.v12i04.5141 \\ R. Tavares, P. Abreu, M. R. Quintas \\ University of Porto, Porto, Portugal
}

\begin{abstract}
The present paper describes a data acquisition wearable device for hand rehabilitation. The main goal of this glove is to be used by patients with hand movement impairment. It has position sensors to measure the bending of synovial joints and sensors to measure the fingertip contact pressure. There is a coin motor and a LED placed on each finger to produce a vibratory and visual stimulus. The glove also tracks the hand rotation and translation using a MPU (Motion Processing Unit) that contains an accelerometer and a gyroscope. A graphical application for an HMI module was developed in order to create rehabilitation game-like exercises where sensor data can be logged for further analysis by a therapist. The wearable device electronic hardware comprises a Glove module and an HMI module that communicate through SPI protocol (Serial Peripheral Interface). The wearable device supports USB connection to send data to a computer or to be used as a peripheral device in virtual or augmented reality applications.
\end{abstract}

Index Terms-Embedded electronics, Hand rehabilitation, Instrumented glove, Wearable monitoring device.

\section{INTRODUCTION}

Stroke is one of the leading causes of death in Europe and hemiparesis affects roughly 80 percent of stroke survivors, causing weakness or movement limitations to one side of the body. The recovery of these movement impairments can be done through frequent stimulation promoted by rehabilitation exercises on a daily basis, taking advantage of the brain plasticity [1].

The rehabilitation of this kind of disorders, such as hemiparesis, usually requires the manipulation of the patient's hand by a specialized therapist and has high associated costs involving daily exercises during several months/years. Some studies show the potential of using haptic devices and instrumented solutions as rehabilitation devices [2]. To accomplish this rehabilitation process, it is necessary to provide devices that enable registering and monitoring the patients' condition. Furthermore, the use of these devices during home rehabilitation promote the success and efficiency of the therapy, lowering the involved costs and countering tedious rehabilitation routines.

\section{WEARABLE REHABILITATION SYSTEM}

The rehabilitation device was developed to promote home exercises required by patients under hand rehabilitation treatments. It uses an instrumented passive glove with a monitoring system capable of recording relevant data that can be further analyzed by the therapist to evaluate

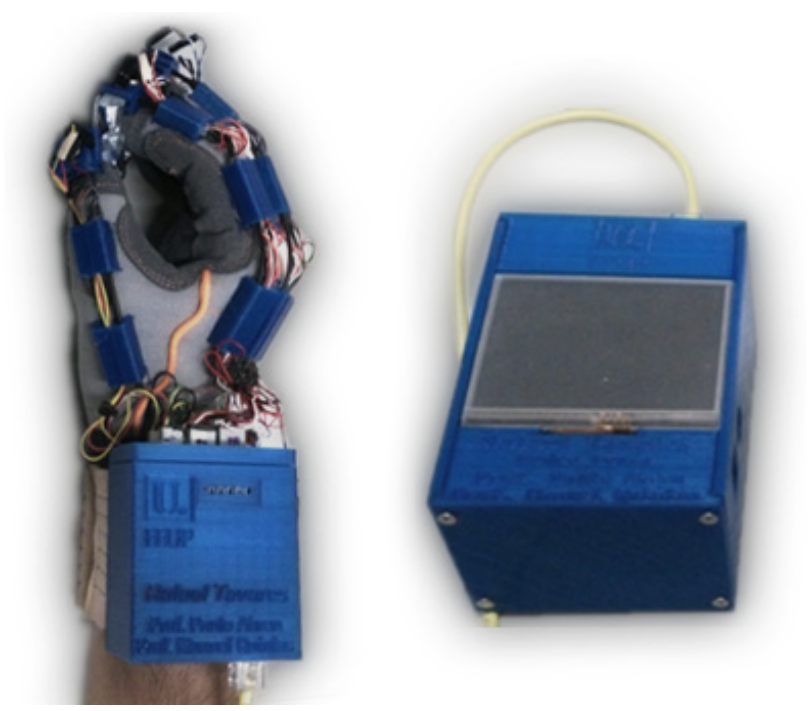

Figure 1. The two models that comprise the system: Glove Module (on the left) and HMI Module (on the right).

the patient's progress. The wearable system can also be used in virtual environment applications.

The system (shown in Fig. 1) comprises two modules for capture, process and display sensor data during rehabilitation exercises [3, 4]:

- Glove Module: A wearable instrumented device fitted with position sensors, fingertip contact pressure sensors, a MPU unit and capable of providing vibrating and visual stimulus. The electronic circuit has a microcontroller with a 12 bits DAC analog sensor data acquisition and supports SPI, $\mathrm{I}^{2} \mathrm{C}$ and USB communication protocols.

- HMI Module: Module having a touchscreen for graphical user interface, with the capability to register data into an external USB storage device and to provide power to the Glove Module.

Fig. 2 shows a schematic representation of the modular architecture of the system, where the main components and the available communications between the modules are presented.

\section{A. Glove Module}

This module uses multiple position sensors (fourteen) to monitor the bending of the fingers. Three position sensors are used for each finger, except for the thumb that uses only two. The adduction-abduction movements and the measurement of the trapeziometacarpal joint (TMC) position were disregarded on this first prototype. The position sensors are housed inside 3D printed components. 


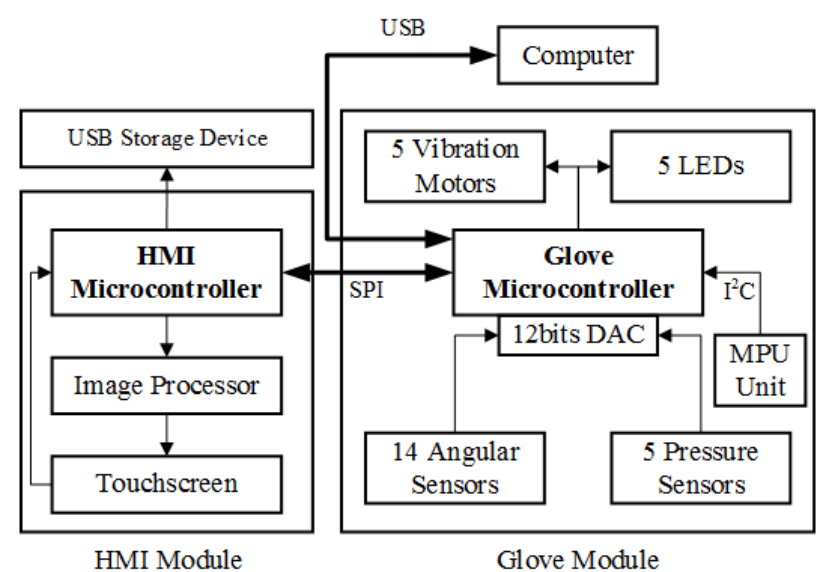

Figure 2. Schematic of the modular system architecture of the rehabilitation system.

There are also five sensors in order to measure the contact pressure on each fingertip. Both position and pressure sensors were developed in-house $[4,5]$. These sensors are based on optical principles and represent an alternative to the solutions that are commonly used on most existing data gloves or rehabilitation devices currently on the market $[6,7]$.

A MPU unit having a combined three-axis accelerometer and three-axis gyroscope is responsible for tracking the hand position and orientation. Each finger also comprises a stimulus system capable of producing vibratory and visual signals, composed by a coin motor and a LED. Since the majority of the rehabilitation exercises rely on movement coordination exercises, this stimulus is used to indicate which finger should be moved.

A dedicated embedded electronic circuit board based on Microchip microcontrollers was built for this module. The microcontroller is responsible to interface with the analog signals from position and pressure sensors, to communicate with the MPU unit through $\mathrm{I}^{2} \mathrm{C}$ protocol, to drive the stimulus system and to communicate with the HMI module.

The modular configuration of the solution provides flexibility for further developments and for integration of new functionalities and sensors into the glove. For example, instead of using the HMI module as an interface, the glove can be connected to a computer through USB to be used as a peripheral device in a virtual or augmented reality application. The developed electronic circuit board shown in Fig. 3 is used for the USB connection between the glove module and a computer.

\section{B. HMI Module}

The HMI module, developed within the work of Sousa [8], is responsible for running the graphical user interface, rehabilitation routines and for logging the exercises. This Microchip microcontroller based embedded circuit board communicates with the Glove module through SPI and also provides the power supply to the glove. Besides running the graphical interface, this module also features data logging to an USB flash drive with separate logs for each event in a csv format file.

\section{HAND Position AND ORIENTATION SENSING}

In an upper limb rehabilitation exercises, the position and orientation of the hand is important for characterizing the dynamics of the patient movements and its evolution.

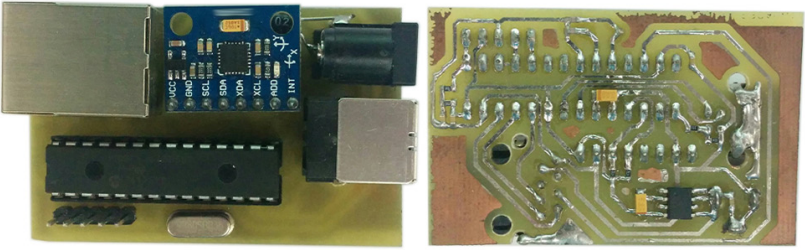

Figure 3. Electronic circuit board for USB connection between the glove module and the computer.

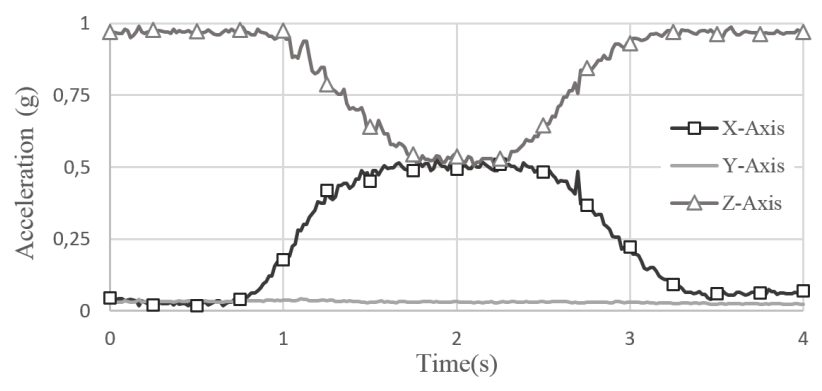

Figure 4. Accelerometer data on each axis during wrist rotation.

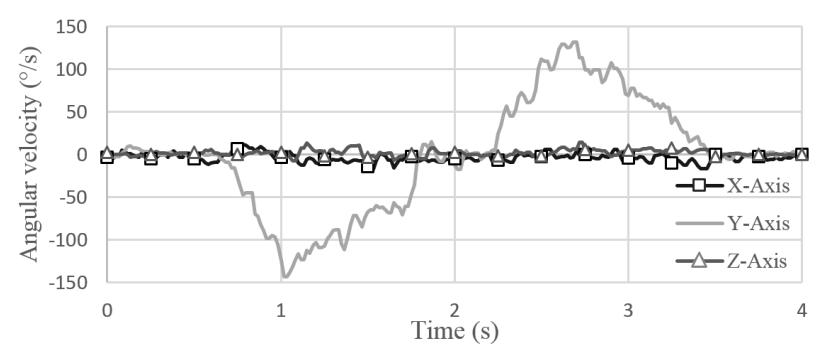

Figure 5. Gyroscope data on each axis during wrist rotation.

For this purpose, a MPU unit was integrated into the wearable device. The selected MPU is a six-axis MEMS motion tracking device from InvenSense, providing the linear and angular acceleration with adjustable scale ranges. The sensor is assembled into the glove and communicates with the microcontroller of the Glove module through $\mathrm{I}^{2} \mathrm{C}$ protocol. The sampling rate and the communication frequency this sensor may provide are compatible with the dynamics required for rehabilitation exercises. The sensor was configured with a sampling rate of 130 $\mathrm{Hz}$.

Tests conducted using the accelerometer with full scale range of $\pm 16 \mathrm{~g}$ and a gyroscope scale range of $\pm 2000 \% \mathrm{~s}$ show that the used sensor ranges do not limit the hand movement recognition.

Fig. 4 and Fig. 5 represent the accelerometer and gyroscope data during a wrist forward-backwards rotation of approximately $45^{\circ}$ of amplitude, starting from a horizontal posture. Since the Y-axis of the MPU sensor is aligned with the arm, the acceleration decreases in the $\mathrm{Z}$ direction and increases in the $\mathrm{X}$ direction in the forward rotation as shown in Fig. 4. Regarding the angular velocity of this movement, it can be seen in Fig. 5 that only the Y-axis velocity has a significant change, being possible to identify the instant $(\mathrm{t} \approx 2 \mathrm{~s})$ the wrist starts the backward rotation.

\section{RESULTS}

A graphical application was developed for data monitoring and rehabilitation exercises. This GUI uses the Microchip Graphics Library functions and was developed 


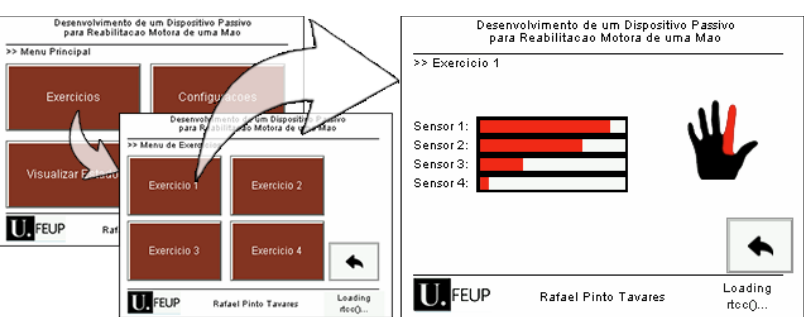

Figure 6. Interface for rehabilitation exercises showing the status of the position $(1,2,3)$ and pressure (4) sensors.

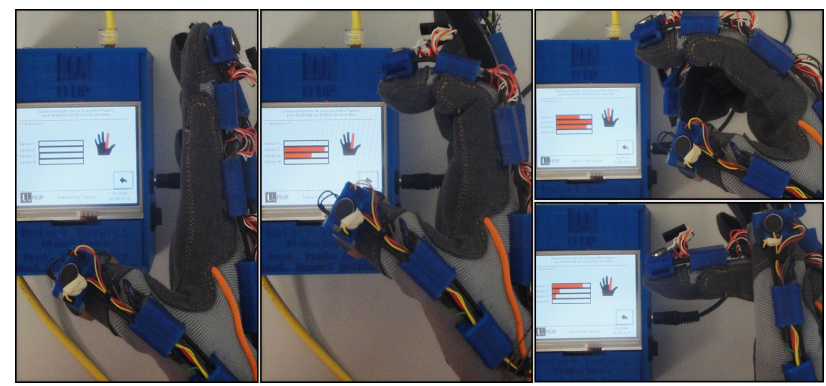

Figure 7. Example of the use of the prototype during a rehabilitation exercise.

with the Graphics Display Designer X (GDDX). It allows the user to monitor each individual sensor, either numerically or with strip charts or bar graphs. Fig. 6 shows some examples of the developed graphical user interface screens that allow the user to monitor the sensors of the selected finger.

Tests were conducted to evaluate the sampling rate of the glove sensors. The system is able to achieve a sampling rate of $50 \mathrm{~Hz}$ that includes the acquisition and data transfer from the glove to the computer.

To evaluate the developed system, an illustrative exercise was implemented. The goal of this exercise is to practice finger coordination, checking the ability of the user to follow a randomly set sequence of movements. During this sequence, the HMI module indicates the finger that should be moved, using a graphical indication complemented with visual and vibratory stimulation. The user can verify the performed movement since the sensor values of the selected finger are shown as a progress bar, as shown in Fig. 7. This goal oriented game-like exercise is an example of what can be implemented with this wearable device to promote the practice of rehabilitation exercises.

\section{CONCLUSIONS}

A functional prototype of a wearable device was built while maintaining low costs. This was possible through the development of embedded electronic circuits based on microcontrollers and using 3D printed components.

The developed wearable device uses position and pressure sensors based on an optical principle that are an alter- native to most common data gloves currently on the market that use resistive or inertial sensor solutions. The developed solution has a MPU sensor for hand position and orientation measurement that uses a sampling rate of 130 $\mathrm{Hz}$.

The software application that runs on the HMI module demonstrates its application in the rehabilitation field through the implementation of game-like exercises. The application allows the recording of relevant information into an USB device to be later analyzed by the therapist. A patient-therapist remote interaction concept can be highly valued as a rehabilitation at home method.

Furthermore, the high potential of the sensor solutions integrated on this glove makes it suitable for other application fields besides rehabilitation, such as finger motion capture on virtual or augmented reality applications, gaming or robotics.

\section{REFERENCES}

[1] D. Jack et al.,"Virtual reality-enhanced stroke rehabilitation" Neural Systems and Rehabilitation Engineering, IEEE Transactions, vol. 9, no. 3, pp. 308-318, Sept 2001. http://dx.doi.org/10.1109/7333.948460

[2] M. Mulas, M. Folgheraiter, and G. Gini, "An EMG-controlled exoskeleton for hand rehabilitation", Rehabilitation Robotics, 2005. ICORR 2005. 9th International Conference on, pp. 371-374, 2005. http://dx.doi.org/10.1109/icorr.2005.1501122

[3] R. Tavares, P. Abreu and M. Quintas, "Instrumented Glove for Rehabilitation Exercises", in $3^{\text {rd }}$ Experiment@International Conference, Ponta Delgada, Portugal, 2015, pp. 107-108.

[4] R. Tavares, "Desenvolvimento de um dispositivo passivo para reabilitação motora de uma mão", Master's thesis, Faculdade de Engenharia da Universidade do Porto, Porto, 2014.

[5] F. Carneiro, "Desenvolvimento de um Sistema de atuação e controlo para dedo de luva de reabilitação", Master's thesis, Faculdade de Engenharia da Universidade do Porto, Porto, 2014.

[6] L. Dipietro, A. M. Sabatini, and P. Dario, "A Survey of GloveBased Systems and Their Applications," Systems, Man, and Cybernetics, Part C: Applications and Reviews, IEEE Transactions on, vol. 38, no. 4, pp. 461-482, Jul. 2008. http://dx.doi.org/10.1109/TSMCC.2008.923862

[7] F. M. Yazadi, M. Schelbert and L. Miller, "Motion capture data glove", U.S. Patent App. 13/192,401, February 2, 2012.

[8] P. Sousa, "Desenvolvimento de um sistema de monitorização low cost de uma câmara frigorífica," Master's thesis, Faculdade de Engenharia da Universidade do Porto, Porto, 2014.

\section{AUTHORS}

R. Tavares is with the INEGI research center, University of Porto, Porto, Portugal (rtavares@inegi.up.pt).

P. Abreu and M. R. Quintas are with the UISPA-LAETA/INEGI research center and with Faculty of Engineering of University of Porto, Porto, Portugal (mrq@fe.up.pt, pabreu@fe.up.pt).

This work was funded by the National Science Foundation (FCT Portuguese Foundation for Science and Technology) under the project UID/EMS/50022/2013. Submitted 13 October 2015. Published as resubmitted by the authors 24 November 2015 . 